\title{
Structural studies on low-dose X-ray radiation induced Transforming growth factor beta-1 (TGF $\beta-1$ ) activation
}

\author{
Timothy R. Stachowski ${ }^{a, b, d}$, Thomas D. Grant ${ }^{a, c}$ and Edward H. Snell ${ }^{a, b}$ \\ a Hauptman Woodward Institute, 700 Ellicott Street, Buffalo, New York 14203, USA, \\ tstachowski@hwi.buffalo.edu

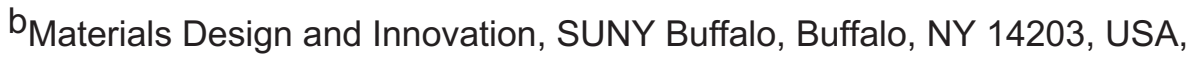 \\ CJacobs School of Medicine and Biomedical Sciences, Structural Biology, SUNY Buffalo, \\ Buffalo, NY 14203, USA, \\ dCell Stress, Roswell Park Comprehensive Cancer Center, Buffalo, NY 14203, USA
}

\begin{abstract}
Understanding the basis of cellular response to radiation is a major goal in the search for effective cancer treatments. Research over the past several years has generated mounting evidence that extracellular signaling proteins orchestrate complicated behaviors between cells that collectively direct the future of tissues, representing a promising new class of targets for biomodulation. Transforming growth factor beta-1 (TGF $\beta-1)$ is of particular interest because in response to radiation damage it initiates downstream signaling pathways that control a number of cancer related processes such as proliferation, migration, and invasion. Normally, the $25 \mathrm{kDa}$ dimer of TGF $\beta-1$ is secreted with the $55 \mathrm{kDa}$ dimer, Latency associated peptide (LAP), that renders TGF $\beta$ 1 inactive, and together are known as Latent-TGF $\beta-1$ (LTGF $\beta-1)$. Dissociation from this arrangement allows the now "activated" TGF $\beta-1$ to bind cognate receptors that initiate signaling pathways and ultimately alter gene expression.
\end{abstract}

X-ray radiation induced TGF $\beta-1$ activation was first observed in the immuno-histochemical staining of irradiated mammary gland cells [1]. This work showed that radiation generated both activated TGF $\beta-1$ and in-activatable (i.e. damaged) LTGF $\beta-1$, suggesting two separate pathways. This was further explored in work showed that reactive oxygen species (ROS) generated in vitro could also activate TGF $\beta$-1 through a non-conserved methionine in LAP [2]. These studies however, overlooked the effect of X-ray generated reductive stress, which could modulate LTGF $\beta$ 1 activity through disulfide disruption, and is a phenomenon that has been observed in other proteins by our lab [3].

Here, we use the tools and knowledge developed in the study of radiation damage in the crystallographic setting to investigate the effects of low dose X-ray radiation exposure on the structural landscape of LTGF $\beta-1$ using small angle X-ray scattering (SAXS) and complementary techniques to (1) characterize changes induced by radiation exposure, (2) determine protein regions most sensitive to radiation, and (3) understand the radiation chemistry that initiates the process. Our results suggest that the damage pathway results from oxidative stress and that activation is initiated but not driven by X-ray exposure. LAP is revealed to be extended when unbound to TGF $\beta-1$. These studies pave the way for the structural understanding of systems impacted by therapeutic level X-ray doses and show the potential impact of radiation damage studies beyond their original intent.

\section{References:}

1. Barcellos-Hoff et al., Molecular Endocrinology,1996

2. Jobling et al., Radiation Research, 2006

3. Sutton et al., Acta Crystallographica Section D,2011 